Radiologe 2021 · 61:1005-1013

https://doi.org/10.1007/s00117-021-00917-0

Angenommen: 27. August 2021

Online publiziert: 28 . September 2021

(c) Springer Medizin Verlag GmbH, ein Teil von Springer Nature 2021

\section{In diesem Beitrag}

- Vorteile und Limitationen der strukturierten Befundung

- Workflow-zentrierte Befundungsinfrastruktur

Hybrid-Reporting - fließender Übergang zwischen Freitext und strukturierter Befundung - Nahtlose Integration der strukturierten Befundung in den klinischen Workflow - Medizinische Inhalte als Kern der strukturierten Befundung • Strukturierte Befundung in der onkologischen Bildgebung am Beispiel des hepatozellulären Karzinoms

- Ausblick

\title{
Integration der strukturierten Befundung in den radiologischen Routine-Workflow
}

\author{
Su Hwan Kim · Sanas Mir-Bashiri · Philipp Matthies · Wieland Sommer . \\ Dominik Nörenberg \\ Smart Reporting GmbH, München, Deutschland
}

\section{Zusammenfassung}

Klinisches/methodisches Problem: Strukturierte Befundung ist seit Jahren eines der meist diskutierten Themen in der Radiologie. Aktuell herrscht ein Mangel an nutzerfreundlichen Softwarelösungen, welche in die bestehende IT-Infrastruktur der Kliniken und Praxen integriert sind und effiziente Dateneingaben erlauben.

Radiologische Standardverfahren: Radiologische Befunde werden meist als Freitext über Spracherkennungssysteme diktiert oder per Tastatur eingegeben. Zudem werden Textbausteine für die Erstellung von Normalbefunden verwendet und bei Bedarf durch Freitextinhalte ergänzt.

Methodische Innovationen: Softwarebasierte Befundungssysteme können Spracherkennungssysteme mit radiologischen Befundvorlagen in Form von interaktiven Entscheidungsbäumen vereinen. Eine technische Integration in RIS(Radiologieinformationssystem)-, PACS(„picture archiving and communication system“)- und AV(, advanced visualization“)-Systeme über Programmierschnittstellen und Interoperabilitätsstandards ermöglicht effiziente Prozesse und die Generierung maschinenlesbarer Befunddaten.

Leistungsfähigkeit: Strukturierte, semantisch annotierte klinische Daten, die über ein strukturiertes Befundungssystem erhoben werden, stehen unmittelbar für epidemiologische Datenauswertungen und kontinuierliches KI(Künstliche Intelligenz)Training zur Verfügung

Bewertung: Der Einsatz der strukturierten Befundung in der radiologischen Routinediagnostik ist mit einer initialen Umstellungsphase verbunden. Eine erfolgreiche Implementierung setzt eine enge Verzahnung der technischen Infrastruktur mehrerer Systeme voraus.

Empfehlung für die Praxis: Durch die Nutzung einer hybriden, softwarebasierten Befundungslösung können radiologische Befunde mit unterschiedlichen Stufen der Struktur generiert werden. Klinische Fragestellungen oder Informationen können aus klinischen Subsystemen semiautomatisch übertragen werden, um vermeidbare Fehler zu eliminieren und die Produktivität zu erhöhen.

\section{Schlüsselwörter}

Befundungssysteme · Künstliche Intelligenz · Befundvorlagen · Strukturierte Datenerfassung . Entscheidungsunterstützung

\section{Vorteile und Limitationen der strukturierten Befundung}

Seit Jahren gibt es zunehmende Bestrebungen internationaler radiologischer Fachgesellschaften, einen einheitlichen Qualitätsstandard für radiologische Befunde zu etablieren und die strukturierte Befundung in die klinische Routine zu in- tegrieren $[7,13,15]$. Diese stützen sich auf robuste Evidenz der Vorteile der strukturierten Befundung in diversen klinischen Anwendungsgebieten. Hierzu zählen u. a. eine verbesserte Befundqualität und -vollständigkeit, eine verbesserte interdisziplinäre Kommunikation sowie eine erhöhte Zufriedenheit der klinischen Zuweiser [1, $3,8,16,21,22,24,28]$. Insbesondere für 


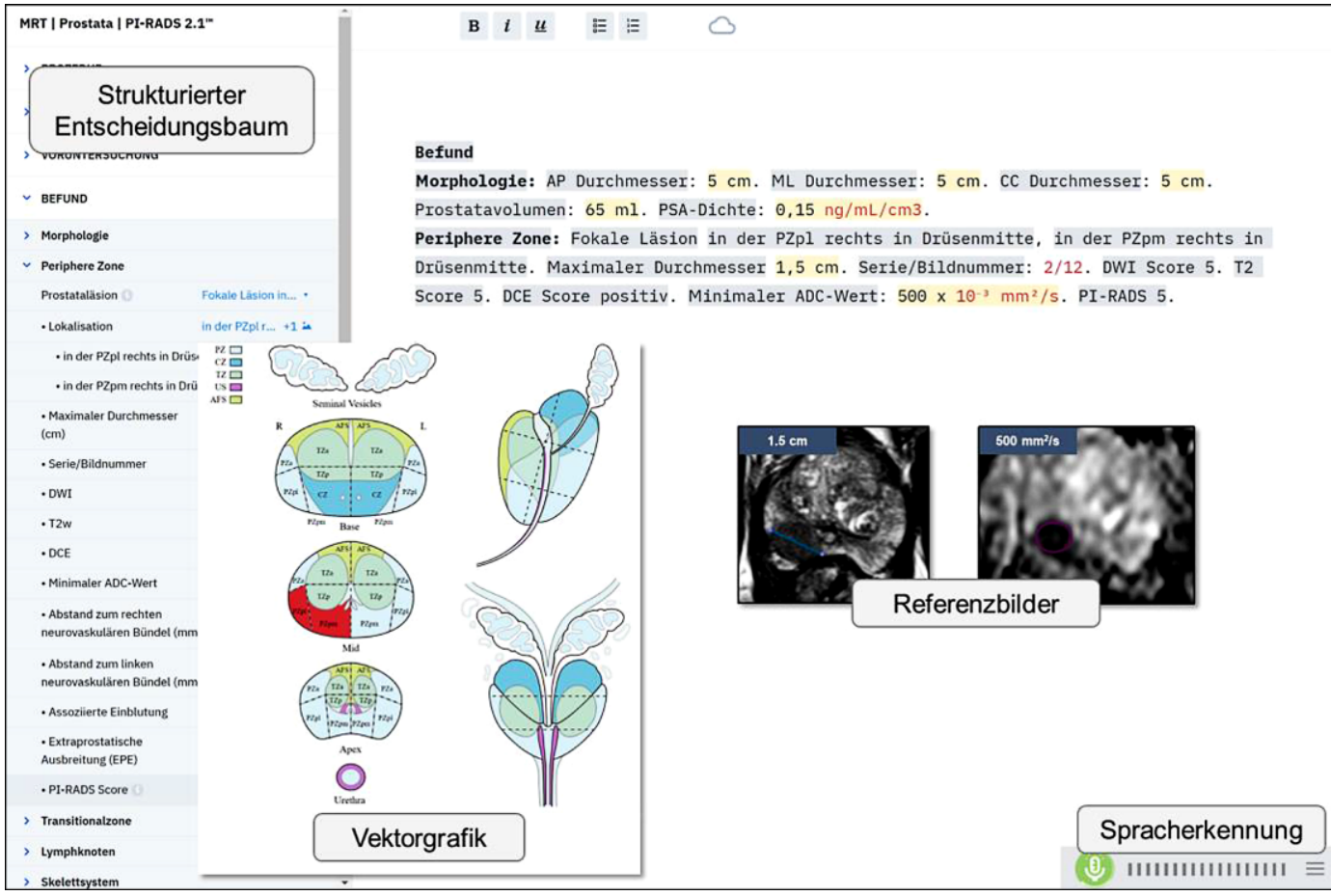

Abb. $1 \triangleleft$ Beispiel einer strukturierten ProstataMRT(Magnetresonanztomographie)-Befundvorlage mit Implementierung der PI-RADS-2.1-Klassifikation und Darstellung von Kernelementen einer softwarebasierten, hybriden Befundungslösung. (Mit freundlicher Genehmigung der Smart Reporting $\mathrm{GmbH}$, München) hochstandardisierte Untersuchungen wie die Magnetresonanztomographie (MRT) des Kopfes bei multipler Sklerose [5] oder der strukturierten onkologischen Befun-

\section{Abkürzungen}

\begin{tabular}{|c|c|}
\hline$A C R$ & American College of Radiology \\
\hline$A P I$ & $\begin{array}{l}\text { Application programming } \\
\text { interface }\end{array}$ \\
\hline AV & Advanced visualization \\
\hline$B I-R A D S$ & $\begin{array}{l}\text { Breast imaging-reporting and } \\
\text { data system }\end{array}$ \\
\hline$B M B F$ & $\begin{array}{l}\text { Bundesministerium für Bildung } \\
\text { und Forschung (BMBF) }\end{array}$ \\
\hline$D R G$ & Deutsche Röntgengesellschaft \\
\hline FHIR & $\begin{array}{l}\text { Fast healthcare interoperability } \\
\text { resources }\end{array}$ \\
\hline HCC & Hepatozelluläres Karzinom \\
\hline LI-RADS & $\begin{array}{l}\text { Liver imaging-reporting and data } \\
\text { system }\end{array}$ \\
\hline KI & Künstliche Intelligenz \\
\hline MRT & Magnetresonanztomographie \\
\hline MRRT & $\begin{array}{l}\text { Management of radiology report } \\
\text { templates }\end{array}$ \\
\hline PACS & $\begin{array}{l}\text { Picture archiving and communi- } \\
\text { cation system }\end{array}$ \\
\hline PI-RADS & $\begin{array}{l}\text { Prostate imaging-reporting and } \\
\text { data system }\end{array}$ \\
\hline RIS & Radiologieinformationssystem \\
\hline RSNA & $\begin{array}{l}\text { Radiological Society of North } \\
\text { America }\end{array}$ \\
\hline SNOMED-CT & $\begin{array}{l}\text { Systematized nomenclature of } \\
\text { medicine-clinical terms }\end{array}$ \\
\hline STRIKE & $\begin{array}{l}\text { Structured therapy planning } \\
\text { with Al decision support for liver } \\
\text { interventions }\end{array}$ \\
\hline
\end{tabular}

dung auf Basis empfohlener Leitlinien wie BI-RADS (Mammographie), PI-RADS (MRT der Prostata) oder LI-RADS (MRT der Leber; $[3,8,23])$ hat sich die strukturierte Befundung als ausgesprochen hilfreich erwiesen. Darüber hinaus sind strukturierte Befunde von überaus großem wissenschaftlichem Interesse, da sie das Potenzial bieten, eine große Menge an maschinenlesbaren strukturierten Daten aus der klinischen Routine zu generieren.

Inzwischen gibt es zahlreiche kommerzielle und nichtkommerzielle Ansätze zur Implementierung der strukturierten Befundung in den klinischen Alltag. Beispielsweise haben radiologische Fachgesellschaften wie die Deutsche Röntgengesellschaft (www.befundung. drg.de) oder die Radiological Society of North America (RSNA) (www.radreport. org) frei zugängliche Sammlungen von Befundvorlagen für eine Vielzahl von klinischen Fragestellungen erstellt und im Online-Format zur Verfügung gestellt.

Doch trotz der zahlreichen Vorteile konnte sich die strukturierte Befundung bislang nicht flächendeckend in der radiologischen Routine durchsetzen [25]. Einer der wichtigsten Gründe hierfür ist die eingeschränkte Workflow-Integration, welche durch unterschiedliche Faktoren bedingt ist: durch häufig unflexible Da- teneingabemethoden (z.B. Mausklicks), wenig granuläre medizinische Inhalte in den Befundvorlagen, eine Einschränkung der Interaktion mit dem Bildviewer sowie die fehlende Einbettung von Befundungslösungen in die bestehende IT-Infrastruktur [27]. Zudem geht die Einführung jeder neuen Methodik zwangsläufig mit einer initialen Umstellungsphase einher, die eine ausführliche Einarbeitung und Änderung von gewohnten Verhaltensweisen im Befundungsprozess erfordert. Vor dem Hintergrund der oft erheblichen Arbeitsbelastung im klinischen Alltag ist nachvollziehbar, dass viele Nutzer neue Befundungslösungen auch bei nur geringsten Produktivitätseinbußen ablehnen und auf den herkömmlichen, sprachgesteuerten Freitextbefund zurückgreifen.

\section{Workflow-zentrierte Befundungs- infrastruktur}

\section{Hybrid-Reporting - fließender Übergang zwischen Freitext und strukturierter Befundung}

Technologische Innovationen können wesentlich dazu beitragen, die strukturierte Befundung effizienter zu gestalten und deren Nutzung in der klinischen Routine zu etablieren [19]. Moderne Softwarelö- 


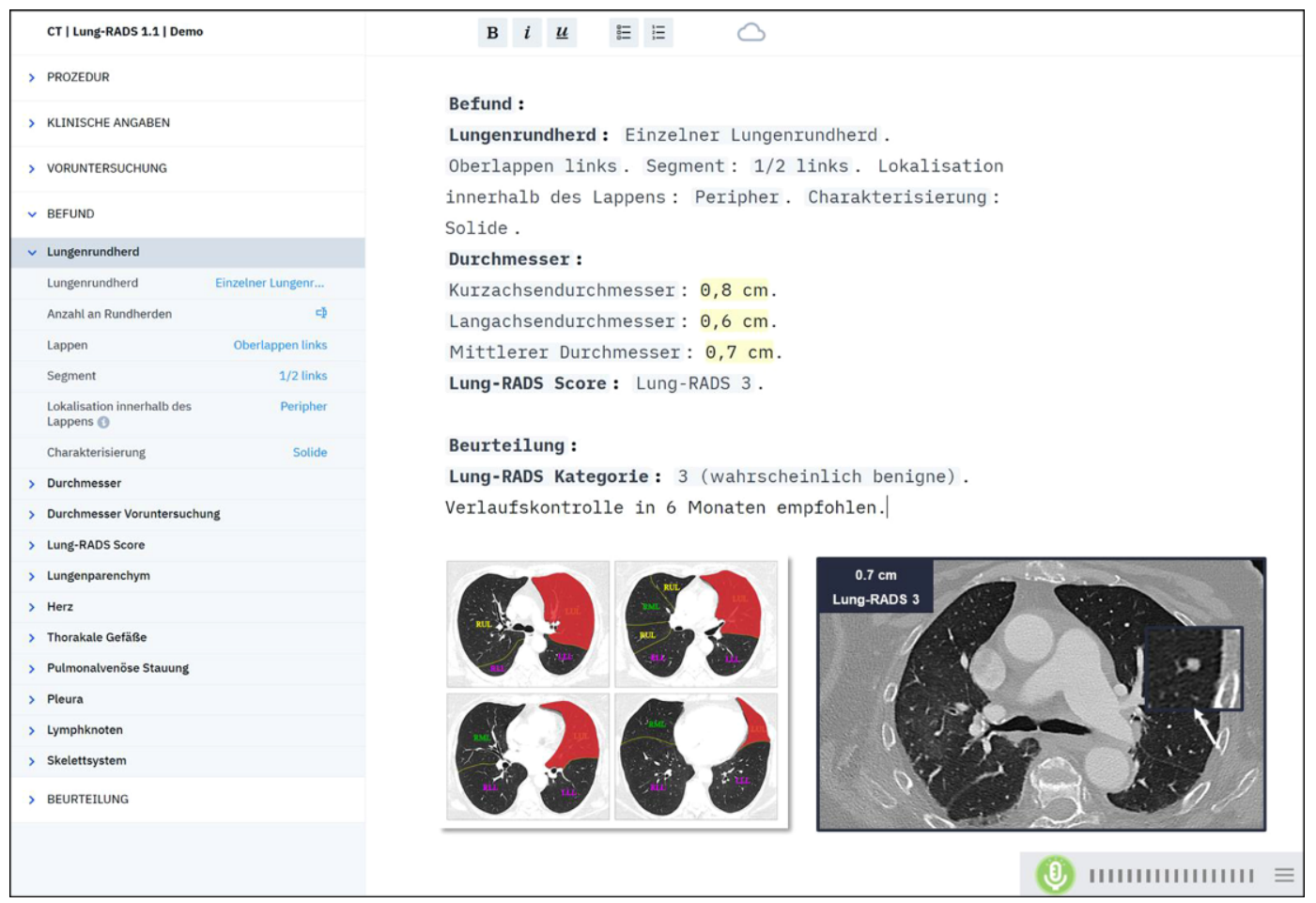

Abb. $2<$ Exemplarischer Multimediabefund mit Implementierung der Computertomographie (CT) LungRADS-1.1-Klassifikation zur strukturierten Befundung von Lungenrundherden mit risikoadaptierter Empfehlung des weiteren Prozederes. (Mit freundlicher Genehmigung der Smart Reporting GmbH, München)

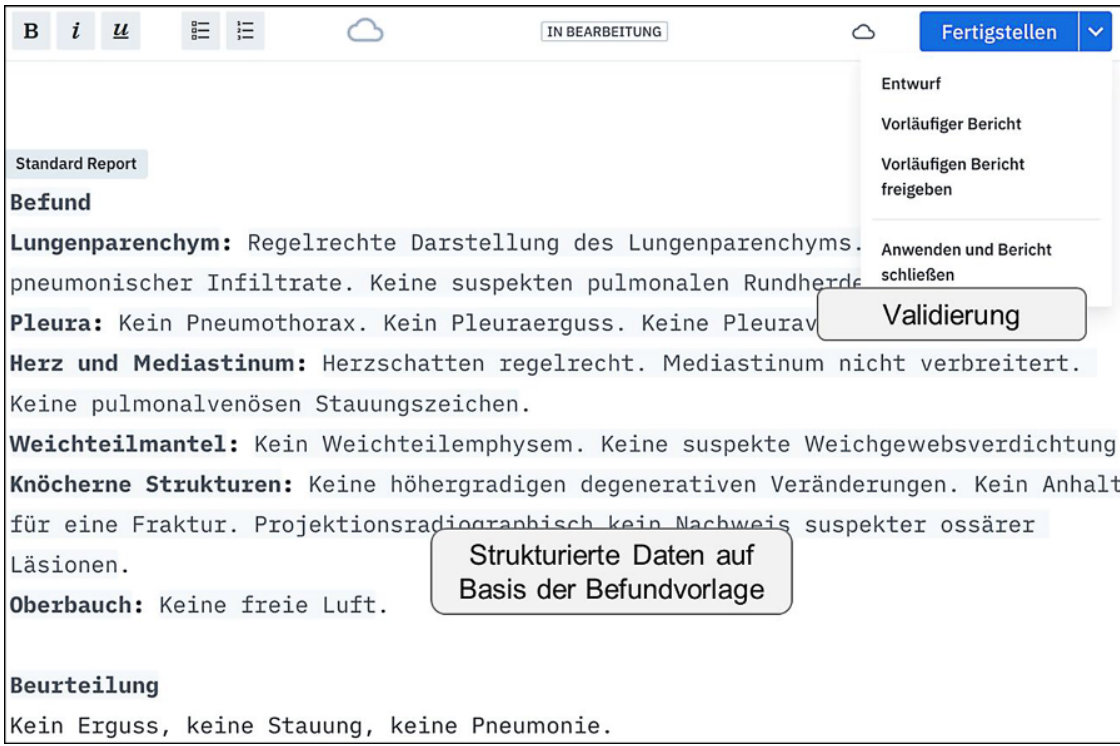

Abb. $3 \Delta$ Hybride Befundungslösung am Beispiel eines Röntgen-Thorax-Befundes. Ein sog. Report Lifecycle ermöglicht eine vollständige fachärztliche Validierung des Befundes zur Qualitätssicherung der generierten, strukturierten Befunddaten. (Mit freundlicher Genehmigung der Smart Reporting $\mathrm{GmbH}$, München)

sungen zur strukturierten Befundung basieren mitunter auf einem hybriden Befundungsansatz, der dem Radiologen maximale Flexibilität einräumen kann und den praktischen Workflow-Anforderungen im klinischen Alltag gerecht wird. Während die meisten herkömmlichen Lösungen dem Radiologen entweder eine ausschließlich klickbasierte, vollständig struk- turierte Befundung oder eine rein unstrukturierte Freitextbefundung zur Auswahl bieten, ermöglichen Hybridsysteme unterschiedlichste Kombinationen dieser beiden Szenarien.

Beispielsweise können strukturierte Befundvorlagen in Form von Entscheidungsbäumen mit einem BefundtextEditor kombiniert werden (-Abb. 1), in dem der Radiologe seinen Befund erstellt. Durch die Kombination von Eingabemasken mit herkömmlichen DateneingabeTools für Freitextinhalte wird der Einstieg in die strukturierte Befundung erleichtert. Insbesondere eine integrierte Spracherkennung fördert die Nutzerfreundlichkeit und Akzeptanz. Um die interdisziplinäre Kommunikation zu fördern und eine eindeutige Befundübermittlung $z u$ gewährleisten, ist die Einbettung von Schlüsselbildern in den finalen Befund entscheidend, um die Planungsgenauigkeit beispielsweise einer Prostatabiopsie basierend auf MRT-Bildern zu erhöhen. - Abb. 1 skizziert exemplarisch einen Multimedia-Befund mit Kernelementen softwarebasierter Befundungslösungen am Beispiel einer Prostata-MRT-Befundvorlage.

Im Ergebnis können durch hybride Befundungslösungen radiologische Befunde mit unterschiedlichsten Stufen der Struktur generiert werden, um die gesamte Bandbreite der klinischen Anwendungsfälle abzudecken - von einem vollständig diktierten Röntgen-Thorax-Befund über die CT-Verlaufskontrolle eines inzidentellen Lungenrundherdes, bei der lediglich der Rundherd anhand von strukturierten Datenelementen beschrieben werden kann, bis hin zu hoch standardisierten mul- 


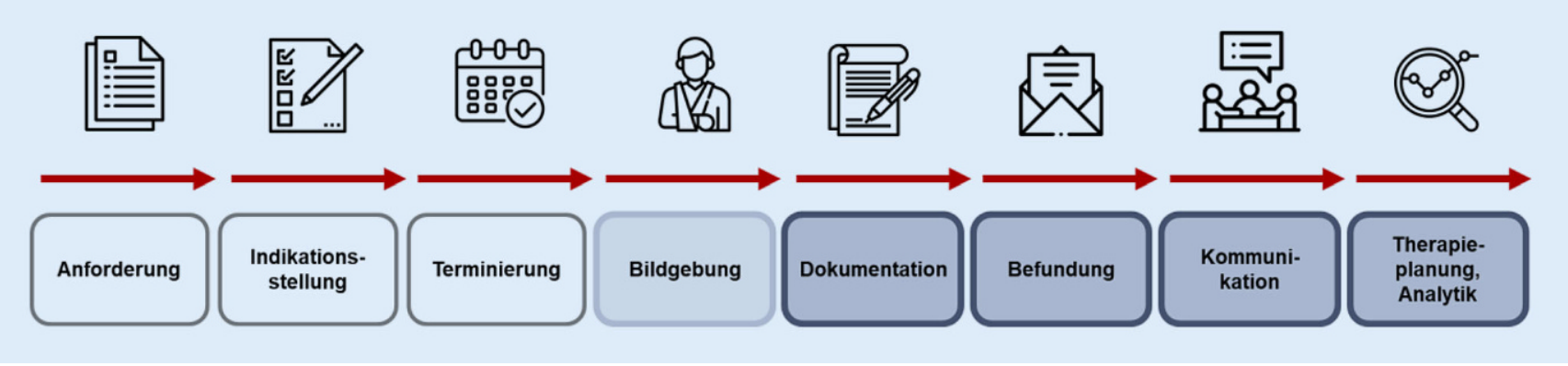

Abb. 4 \ Übersicht des radiologischen Workflows. Nach der Untersuchungsanforderung durch den zuweisenden Arzt ist die Indikationsstellung durch den Radiologen erforderlich, gefolgt von der Terminierung des Patienten. Im Anschluss an die Bildgebung erfolgt die Dokumentation und Befundung der radiologischen Bilder sowie die Kommunikation der Befundergebnisse, welche als wichtige Grundlage für die weiteren Therapieentscheidungen dienen. Die (Teil-)Automatisierung dieser Prozesskette mit Reduktion redundanter Arbeitsschritte soll zunehmend durch Softwareunterstützung und Methoden der künstlichen Intelligenz den klinischen Workflow effizienter gestalten. (Diese Abbildung wurde erstellt mithilfe von Ressourcen von Flaticon.com)

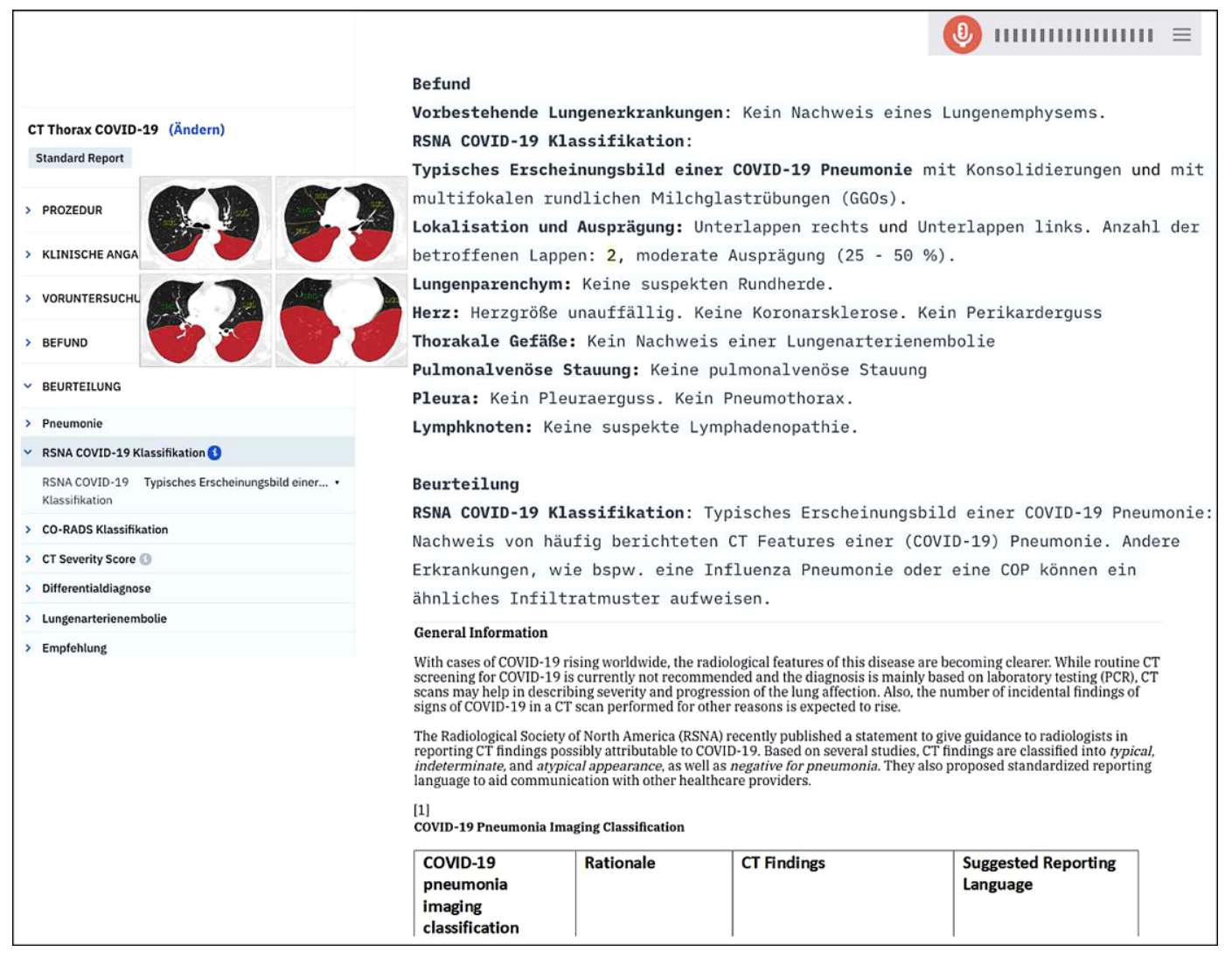

Abb. $5 \varangle$ Strukturierte Befundvorlage einer Computertomographie (CT) des Thorax (COVID-19, "corona virus disease $19^{\prime \prime}$ ) basierend auf der RSNA-COVID19-Klassifikation inklusive klinischer Hintergrundinformationen (unterer Teil). (Mit freundlicher Genehmigung der Smart Reporting $\mathrm{GmbH}$, München) tiparametrischen Prostata-MRT-Befunden gemäß der PI-RADS-2.1-Klassifikation ([17, 29]; - Abb. 1). Diese Flexibilität senkt zudem die Hürde der abteilungsweiten Einführung einer solchen Befundungslösung maßgeblich, da die Einführung eines strukturierten Befundungsansatzes stufenweise erfolgen kann und der gewohnte, sprachgesteuerte Workflow bei Bedarf jederzeit zur Verfügung steht.
Ein weiterer großer Vorteil von strukturierten Befundvorlagen ist die Möglichkeit, die Befundinhalte anhand einheitlicher Kriterien zu standardisieren, die etwa von Leitlinien und Fachgesellschaften vorgegeben werden. Dies ermöglicht es den Zuweisern, die in dem Befund enthaltenen Informationen besser zu verstehen und deren Implikationen in einer konsistenten Weise für die weitere klinische Entscheidungsfindung zu nutzen, unabhän- gig davon, welcher Radiologe den Befund verfasst hat. Ein bekanntes Beispiel für die kriterienbasierte Lungenrundherddiagnostik ist die Lung-RADS-Klassifikation ([14, 30]; - Abb. 2). 


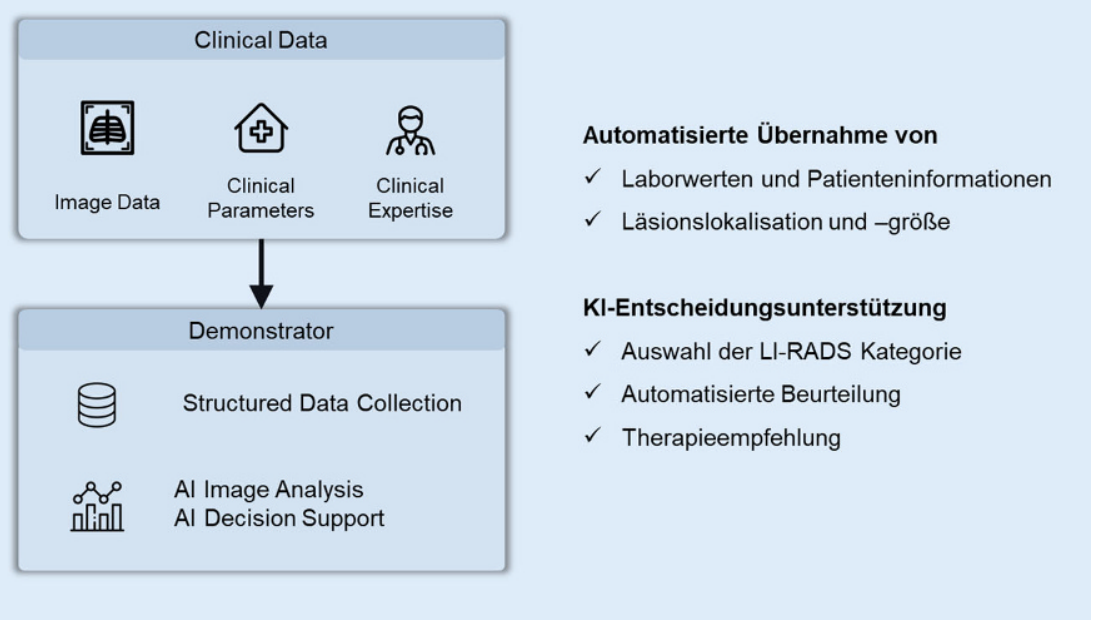

Abb. 6 \ Schematische Übersicht eines Demonstrators, der Bildanalysen durch künstliche Intelligenz (KI) sowie ein KI-Entscheidungsunterstützungssystem in strukturierte Befundvorlagen integriert, um die Therapieauswahl und -planung für das hepatozelluläre Karzinom (HCC) zu unterstützen. (Diese Abbildung wurde erstellt mithilfe von Ressourcen von Flaticon.com)

Nahtlose Integration der strukturierten Befundung in den klinischen Workflow

Viele webbasierte Befundungslösungen sind von klinischen Informationssystemen wie dem Radiologieinformationssystem (RIS), dem Picture Archiving and Communication System (PACS) und von Advanced Visualization (AV) Tools vollständig getrennt, sodass relevante Informationen nicht von und zu diesen Applikationen kommuniziert werden können.

Diese technischen Limitationen in der praktischen Anwendung schränken die Effizienz im klinischen Arbeitsalltag ein. Moderne Befundungslösungen adressieren diese Anforderungen durch eine tiefe technische Integration in klinische RIS-, PACS- und AV-Systeme über standardisierte Programmierschnittstellen (API; „application programming interface ${ }^{\prime \prime}$ ). Hierbei werden Interoperabilitätsstandards wie HL7 v2 oder FHIR (,fast healthcare interoperability resources") verwendet, um konsistente Daten-Kommunikationsprotokolle zu definieren [2].

Häufig wird zwischen einem PACSgesteuerten und einem RIS-gesteuerten radiologischen Workflow unterschieden. Diese Begrifflichkeiten beschreiben, von welchem System aus die radiologische Arbeitsliste gestartet und navigiert wird $[9,10]$. Interoperable Befundungssysteme lassen sich in beide Arten des Workflows integrieren, sodass der Befund-Editor aus dem jeweiligen System geöffnet werden kann. Die Effizienz kann noch weiter gesteigert werden, indem durch die vom RIS- oder PACS-System übermittelten Angaben zur Modalität und Körperregion relevante Befundvorlagen gefiltert oder sogar automatisch ausgewählt werden.

Für die eigentliche Befunderstellung bedeutet eine tiefe Integration, dass klinische Fragestellungen, prozedurale Informationen und Dosisberichte automatisch aus dem RIS oder PACS in den Befund übernommen werden können. Ebenso können Messparameter aus dem PACSoder AV-System - etwa die Durchmesser von Lymphknoten oder Lungenrundherden - bei Annotation der radiologischen Bilder im DICOM-Viewer unmittelbar auf die entsprechenden Datenfelder im strukturierten Befund übertragen werden. Hierdurch ist das händische Kopieren und Einfügen von Informationen zwischen Systemen nicht mehr erforderlich, wodurch Übertragungsfehler eliminiert werden können. Nach Erstellung des ersten Entwurfes können mithilfe des sog. Report Lifecycles sämtliche Validierungsschritte des radiologischen Befundes bis hin zur fachärztlichen Freigabe innerhalb des Befundungssystems erfolgen, um eine vollständige Qualitätssicherung der generierten strukturierten Befunddaten zu gewährleisten (•Abb. 3). Der initiale Befundentwurf kann hierbei flexibel über eine Änderung der strukturierten Parameter oder durch Freitextbearbeitungen angepasst werden. Die unterschiedlichen Befundversionen werden abgespeichert, sodass die Änderungen auch nachträglich nachvollziehbar sind.

Durch die Formatierung mit Logos, Kopf- und Fußnoten sowie Signaturen können schließlich formale, institutsspezifische Befunddokumente generiert und beispielsweise an das RIS gesendet werden. Der finale Befunddatensatz kann je nach Bedarf in einem strukturierten (z.B. JSON) oder unstrukturierten (z.B. „plain text", PDF) Dateiformat exportiert werden und damit für nachfolgende Auswertungen genutzt werden. Durch die strukturierte Generierung des radiologischen Befundes kann eine semantische Annotation von Befunddaten durch standardisierte Terminologien erfolgen, sodass die Befunddaten für eine maschinelle Interpretation zur Verfügung stehen. Unter den internationalen Terminologien, die zur Annotation genutzt werden, ist im Bereich der Radiologie das von der RSNA entwickelte RadLex führend, während SNOMED CT (,systematized nomenclature of medicine-clinical terms ${ }^{\prime \prime}$ ) im Allgemeinen als umfangreichste klinische Terminologie gilt.

In Abb. 4 ist eine Übersicht des radiologischen Workflows dargestellt [12], wobei insbesondere eine zunehmend automatisierte Befunderstellung und -kommunikation künftig zur Effizienzsteigerung und verbesserten Therapieplanung beitragen können.

\section{Medizinische Inhalte als Kern der strukturierten Befundung}

Zahlreiche Studien haben in der Vergangenheit den positiven Einfluss der strukturierten Befundung auf die Befundqualität und -vollständigkeit belegt. Jedoch sind Struktur und Inhalt der Befunde unmittelbar abhängig vom Aufbau der genutzten Befundvorlagen (Templates). Diese bestehen häufig aus unterschiedlichen Auswahl- und Eingabeelementen. Gemäß der IHE MRRT-Profile („management of radiology report templates") bestehen die gängigen Befundvorlagen meist aus den Sektionen "Prozedur", "Klinische Informationen", "Voruntersuchung", "Befund" und 


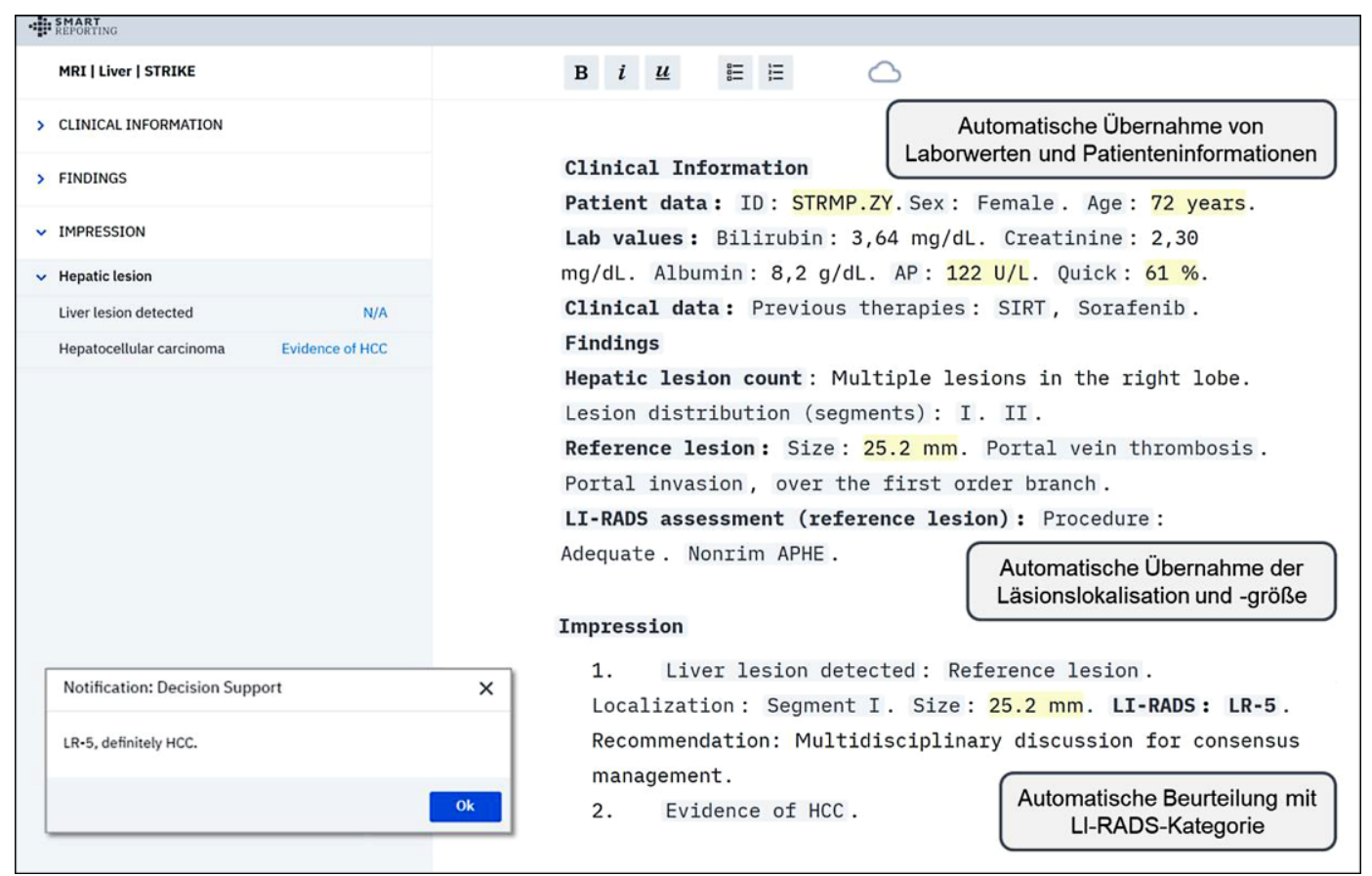

Abb. 7 A Prototypischer Demonstrator als Teil des wissenschaftlichen Kooperationsprojekts STRIKE („structured therapy planning with Al decision support for liver interventions"; gefördert durch das Bundesministerium für Bildung und Forschung, $\mathrm{BMBF}$ ). Der Demonstrator umfasst eine strukturierte Befundvorlage zur Befundung von hepatozellulären Karzinomen (HCC) nach den LI-RADS-Kriterien (v2018) mit integrierter Entscheidungsunterstützung durch automatische Vorauswahl der LIRADS-Kategorie basierend auf den im Entscheidungsbaum angewählten Elementen. (Mit freundlicher Genehmigung der Smart Reporting $\mathrm{GmbH}$, München)

„Beurteilung“. Für den praktischen Nutzen ist eine optimale Balance hinsichtlich der Granularität entscheidend. Eine zu schlichte Befundvorlage kann keinen besonderen Mehrwert zur Standardisierung und Qualitätsverbesserung leisten, wohingegen ein zu detailliertes Template mit mehrfach verschachtelten Elementen schwierig zu bedienen sein kann.

Das in • Abb. 5 skizzierte Template zur Befundung einer CT des Thorax mit der Frage nach einer COVID-19-Erkrankung veranschaulicht einen solchen balancierten Aufbau, der eine detaillierte Beschreibung von COVID-19-relevanten Elementen basierend auf der RSNA-Klassifikation erlaubt [26], wohingegen die restlichen Inhalte vergleichsweise einfach strukturiert sind.

Radiologische Befundvorlagen sollten unter Beteiligung radiologischer Fachärzte und Fachgesellschaften unter Berücksichtigung aktueller Leitlinien erstellt werden und einem mehrstufigen Review-Prozess unterliegen. Das Einbetten didaktisch wertvoller Inhalte und Kriterien zur Entscheidungsunterstützung (wie z. B. klickbare Grafik-Elemente zur erleichterten Angabe anatomischer Lokalisationen, Klassi- fikationen und Beispielbilder für pathologische Bildbefunde in Form von Infoboxen) unterstützen die Workflow-Effizienz, sodass Inhalte nicht separat in OnlineSuchmaschinen oder Lehrbüchern nachgeschlagen werden müssen (• Abb. 5).

Strukturierte Befundung in der onkologischen Bildgebung am Beispiel des hepatozellulären Karzinoms

In den letzten Jahren hat sich die strukturierte Befundung insbesondere in der onkologischen Bildgebung etabliert, was im Folgenden am Beispiel des hepatozellulären Karzinoms (HCC) verdeutlicht wird. In einer Studie von Peter et al. konnte gezeigt werden, dass die Anwendung einer strukturierten Befundvorlage zu einer signifikant erhöhten Detektion von HCCsuspekten Leberläsionen geführt hat [20]. Aufgrund der unterschiedlichen Indikationsspektren sowie der Komplexität der verfügbaren Therapieoptionen stellt die Therapieplanung für HCC eine große Herausforderung dar. Um dieser Herausforderung zu begegnen, können bildgebende
Parameter quantitativ erfasst und mit klinischen Parametern zusammengeführt werden. Darüber hinaus sind zunehmend KIgestützte Ansätze zur optimierten Bildinterpretation und Therapieplanung in den Fokus gerückt. Abb. 6 und 7 zeigen exemplarisch die Übersicht eines Demonstrators für die bild- und befundgestützte Tumorcharakterisierung und Therapieplanung des HCC als Teil des wissenschaftlichen Kooperationsprojekts STRIKE („structured therapy planning with Al decision support for liver interventions"; [31]).

Im Detail nutzt der Demonstrator dabei Entscheidungsbäume aus strukturierten Befundvorlagen, um klinische Daten der Patienten und quantitative Parameter aus Bilddaten strukturiert zu erfassen. Diese Daten werden mit der aktuellen Literatur (wissenschaftliche Publikationen, Leitlinien) abgeglichen und in einen Therapievorschlag umgesetzt.

Die STRIKE-Befundvorlage des Demonstrators beruht auf den vom ACR herausgegebenen LI-RADS Guidelines (v2018; [31]) und ermöglicht eine automatische Vorauswahl der LI-RADS-Kategorie anhand der Selektionen im Strukturbaum 


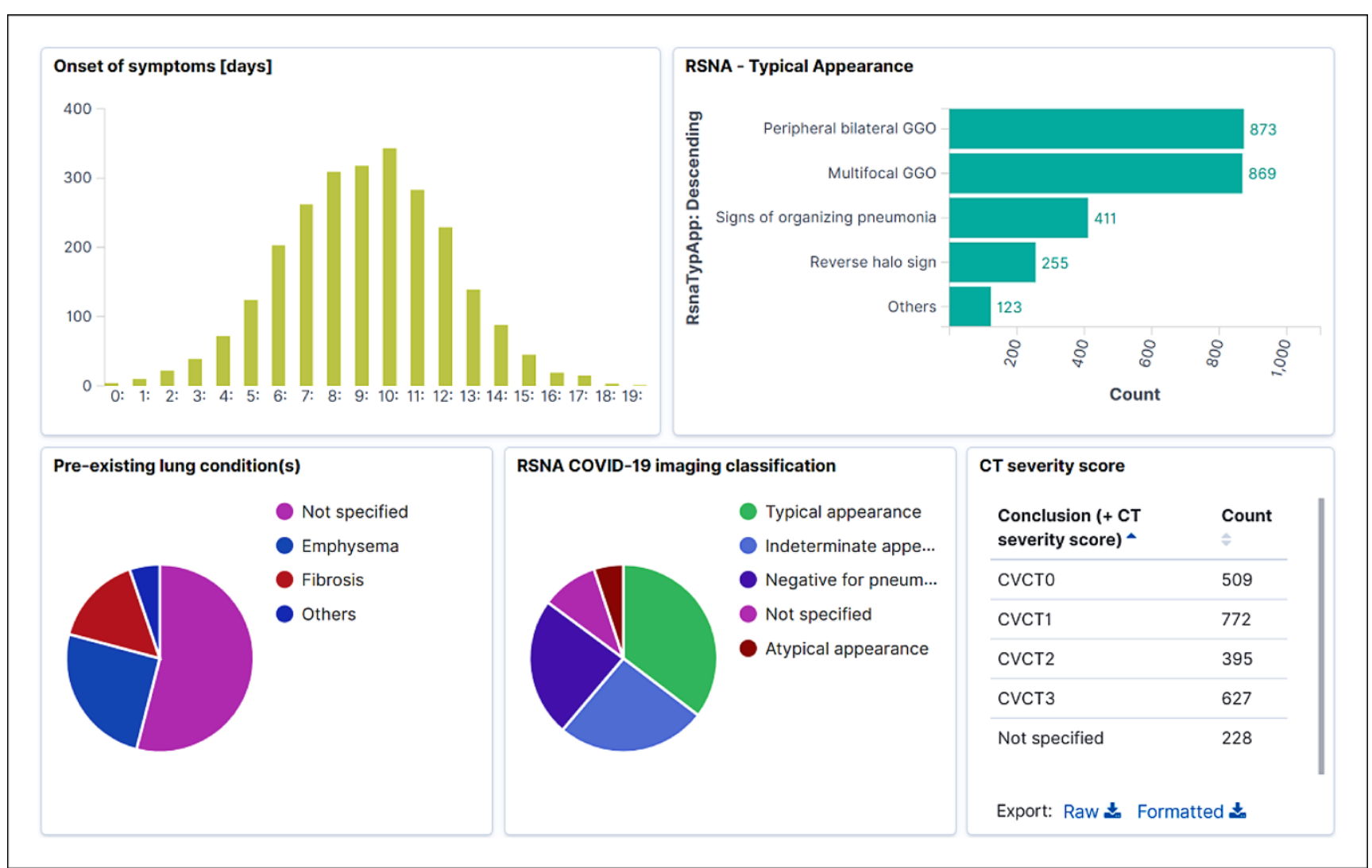

Abb. 8 ॥ Echtzeit-Dashboard zur Visualisierung von Befunddaten aus strukturierten computertomographischen COVID-19Thoraxbefunden

(- Abb. 7). Darauf aufbauend wird zudem ein Vorschlag zu Verlaufsuntersuchungen und möglichen Behandlungsoptionen gegeben. Ein holistischer Ansatz einer automatisierten Tumorcharakterisierung ist auf alle Tumorentitäten übertragbar und stellt neue Möglichkeiten zur Realisierung der Präzisionsmedizin in Aussicht.

\section{Ausblick}

In dem vorliegenden Artikel wurde die enorme Bedeutung der nahtlosen Workflow-Integration für die Akzeptanz und Nutzung strukturierter Befundungslösungen durch Radiologen aufgezeigt. Für den einzelnen befundenden Arzt dient die standardisierte und strukturierte Dokumentation vor allem als Tool zur Orientierungshilfe, Qualitätssteigerung und effizienteren Befundung.

In den kommenden Jahren ist eine zunehmende Automatisierung von Befundungsprozessen zu erwarten, etwa durch eine direkte Vorbefüllung des strukturierten Befundes durch Messwerte aus den Bildviewern sowie von Kl-gestützten Bildanalyse-Algorithmen und Entscheidungsunterstützungssystemen [4, 6]. Ein Beispiel für die KI-basierte Integration von Bilddaten in strukturierte Befunde ist der CAD4COVID-CT-Prototyp zur CT-ThoraxBefundung einer COVID-19-Erkrankung, der COVID-19-spezifische KI-Ergebnisparameter (z.B. dem Schweregrad der pulmonalen Verdichtungen) automatisch in einen strukturierten Befund einfügt (https://smartcad.smart-radiology.com). Entsprechende Algorithmen eignen sich beispielsweise für eine KI-basierte Risikostratifizierung von COVID-19-Patienten [11].

Zudem wird es zukünftig entscheidend sein, immer intelligentere Prozesse für die Befundung zu entwickeln. Da immer mehr Fachgesellschaften Empfehlungen für strukturierte Inhalte erstellen und die Anzahl der Klassifikationssysteme jährlich wächst, wird es für den Radiologen unmöglich, jedes Klassifikationssystem zu kennen und das richtige Lexikon zur Beschreibung eines Befundes zu verwen- den. Computergestützte Anwendungen können Radiologen bei der Befundung helfen, indem sie - etwa auf der Grundlage von Kontextinformationen aus dem elektronischen Krankenaktensystem und der Bildgebungsstudie - potenziell relevante Klassifizierungssysteme oder modulare medizinische Inhalte empfehlen.

Neben der Standardisierung und Automatisierung von klinischen Prozessen gewinnt im Sinne der Präzisionsmedizin der Wunsch nach einer datengestützten Entscheidungsfindung an Bedeutung. Dadurch, dass strukturierte, semantisch annotierte klinische Befunddaten im Gegensatz zu unstrukturierten Freitextinhalten maschinenlesbar sind, stehen diese unmittelbar für Datenauswertungen, kontinuierliches $\mathrm{KI}$-Training und personalisierte Therapieentscheidungen zur Verfügung [18]. Die klinischen Daten, die während der strukturierten Befundung in Echtzeit generiert werden, können beispielsweise mithilfe interaktiver Dashboards visualisiert werden (• Abb. 8). 
Es ist damit zu rechnen, dass für eine großflächige Auswertbarkeit dieser überaus wertvollen strukturierten Befunddaten, die idealerweise qualitätskontrolliert und fachärztlich validiert in der klinischen Routine erhoben werden, eine abteilungsund institutionsübergreifende Verlinkung und Aggregation von Daten aus weiteren Informationssystemen in Zukunft eine entscheidende Rolle spielen wird. Nicht zuletzt ist der Ansatz zur strukturierten medizinischen Dokumentation und Datensammlung auf weitere klinische Fachbereiche, wie z. B. die Pathologie oder Onkologie, übertragbar.

\section{Fazit für die Praxis}

- Durch die Nutzung einer hybriden Befundungslösung können radiologische Befunde mit unterschiedlichen Stufen der Struktur generiert und in den klinischen Workflow integriert werden, womit die gesamte Bandbreite klinischer Anwendungsfälle abgedeckt wird.

- Die Integration strukturierter Befundungssysteme in klinische RIS(Radiologieinformationssystem)-, PACS(„picture archiving and communication system ")- und AV (,,advanced visualization“)-Systeme ermöglicht deren Etablierung und Nutzung in der radiologischen Routinediagnostik zur Erhebung von maschinenlesbaren Real-World-Daten.

- Klinische Fragestellungen oder prozedurale Informationen können aus klinischen Subsystemen semiautomatisch in einen Befundtext-Editor übertragen werden, sodass strukturierte Befundvorlagen zur Prozessautomatisierung beitragen und semantische Daten generieren, die für KITraining genutzt werden können.

- Strukturierte Befundungssysteme stellen eine Plattform für die Integration von KI(künstliche Intelligenz)-Lösungen zur Bilddatenanalyse dar.
Korrespondenzadresse

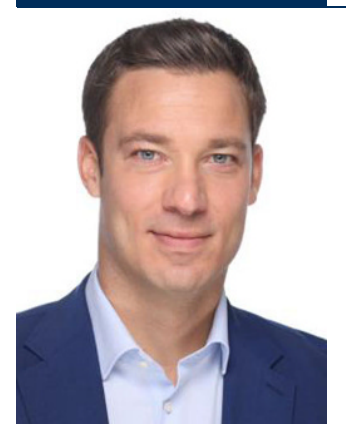

PD Dr. med. Dominik Nörenberg

Smart Reporting $\mathrm{GmbH}$

Brienner Straße 11-13, 80336 München,

Deutschland

d.noerenberg@smart-reporting.com

Danksagung. Das Forschungsprojekt "STRIKE“ des Bundesministeriums für Bildung und Forschung (BMBF) wird im Rahmen von „Bildgeführte Diagnostik und Therapie - Neue Wege in der Intervention" unter dem Förderkennzeichen 13GW0369A multizentrisch mit Partnern aus Universitäten, Forschungseinrichtungen und Industrie gefördert. Der KI-Prototyp CAD4COVID wurde in Zusammenarbeit der Smart Reporting GmbH mit dem niederländischen Unternehmen Thirona entwickelt und steht öffentlich für wissenschaftliche Auswertungen zur Verfügung. Das Forschungsvorhaben wurde zudem von der European Open Science Cloud (EOSC) der Europäischen Kommission gefördert.

\section{Einhaltung ethischer Richtlinien}

Interessenkonflikt. S.H. Kim, S. Mir-Bashiri, P. Matthies, W. Sommer und D. Nörenberg geben an, dass ein Interessenkonflikt besteht, da sie von der Firma Smart Reporting $\mathrm{GmbH}$ angestellt sind.

Für diesen Beitrag wurden von den Autoren keine Studien an Menschen oder Tieren durchgeführt. Für die aufgeführten Studien gelten die jeweils dort angegebenen ethischen Richtlinien.

\section{Literatur}

1. Armbruster $M$, Gassenmaier $S$, Haack $M$ et al (2018) Structured reporting in petrous bone MRI examinations: impact on report completeness and quality. Int J Comput Assist Radiol Surg. https://doi. org/10.1007/s11548-018-1828-1

2. Bauer J, Rohner-Rojas S, Holderried M (2019) Einrichtungsübergreifende Interoperabilität. Radiologe 60:334-341. https://doi.org/10.1007/ S00117-019

3. Brook OR, Brook A, Vollmer CM et al (2015) Structured reporting of multiphasic $\mathrm{CT}$ for pancreatic cancer: potential effect on staging and surgical planning. Radiology 274:464-472. https:// doi.org/10.1148/radiol.14140206

4. Cuocolo R, Caruso M, Perillo Tet al (2020) Machine learning in oncology: a clinical appraisal. Cancer Lett 481:55-62. https://doi.org/10.1016/j.canlet. 2020.03.032
5. Dickerson E, Davenport MS, Syed F et al (2017) Effect of template reporting of brain MRis for multiple sclerosis on report thoroughness and neurologist-rated quality: results of a prospective quality improvement project. J Am Coll Radiol 14:371-379. https://doi.org/10.1016/j.jacr.2016. 09.037

6. Erickson BJ, Korfiatis P, Akkus Z, Kline TL (2017) Machine learning for medical imaging. Radiographics 37:505-515. https://doi.org/10.1148/rg. 2017160130

7. European Society of Radiology (2011) Good practice for radiological reporting. Guidelines from the European Society of Radiology (ESR). Insights Imaging 2:93-96. https://doi.org/10. 1007/s13244-011-0066-7

8. Flusberg M, Ganeles J, Ekinci T et al (2017) Impact of a structured report template on the quality of CT and MRI reports for hepatocellular carcinoma diagnosis. J Am Coll Radiol 14:1206-1211. https:// doi.org/10.1016/j.jacr.2017.02.050

9. Forsberg D, Rosipko B, Sunshine JL, Ros PR (2016) State of integration between PACS and other IT systems: a national survey of academic radiology departments. J Am Coll Radiol 13:812-818.e2. https://doi.org/10.1016/J.JACR.2016.01.018

10. Geeslin MG, Gaskin CM (2016) Electronic health record-driven Workflow for diagnostic radiologists. J Am Coll Radiol 13:45-53. https://doi.org/10. 1016/J.JACR.2015.08.008

11. Gresser E, Reich J, Sabel BO et al (2021) Risk stratification for ECMO requirement in COVID-19 ICU patients using quantitative imaging features in CT scans on admission. Diagnostics 11:1029. https://doi.org/10.3390/diagnostics11061029

12. Hackländer T (2013) Strukturierte Befundung in der Radiologie. Radiologe 53:613-617. https://doi. org/10.1007/S00117-013-2493-6

13. Kalra G, Bhugra DK, Shah N et al (2018) ESR paper on structured reporting in radiology. Insights Imaging 9:1-7. https://doi.org/10.1007/s13244017-0588-8

14. Martin MD, Kanne JP, Broderick LS et al (2017) Lung-RADS: pushing the limits. Radiographics 37:1975-1993. https://doi.org/10.1148/ rg.2017170051

15. Morgan TA, Helibrun ME, Kahn CE (2014) Reporting initiative of the radiological society of North America: progress and new directions. Radiology 273:642-645. https://doi.org/10.1148/radiol. 14141227

16. Nörenberg $D$, Sommer $W H$, Thasler $W$ et al (2017) Structured reporting of rectal magnetic resonance imaging in suspected primary rectal cancer: potential benefits for surgical planning and interdisciplinary communication. Invest Radiol 52:232-239. https://doi.org/10.1097/RLI. 0000000000000336

17. Padhani AR, Barentsz J, Villeirs G et al (2019) PIRADS steering committee: the PI-RADS multiparametric MRI and MRI-directed biopsy pathway. Radiology 292:464-474. https://doi.org/10.1148/ radiol.2019182946

18. Pinto dos Santos D, Baeßler B (2018) Big data, artificial intelligence, and structured reporting. Eur RadiolExp 2:1-5. https://doi.org/10.1186/s41747018-0071-4

19. Dos PSD, Hempel JM, Mildenberger P et al (2019) Structured reporting in clinical routine. RoFo 191:33-39. https://doi.org/10.1055/a-0636-3851

20. Poullos PD, Tseng JJ, Melcher ML et al (2018) Structured reporting of multiphasic CT for hepatocellular carcinoma: effect on staging and suitability for transplant. Am J Roentgenol 
210:766-774. https://doi.org/10.2214/AJR.17. 18725

21. Sabel BO, Plum JL, Kneidinger $N$ et al (2017) Structured reporting of $\mathrm{CT}$ examinations in acute pulmonary embolism. J Cardiovasc Comput Tomogr 11:188-195. https://doi.org/10.1016/j. jcct.2017.02.008

22. Sahni VA, Silveira PC, Sainani NI, Khorasani R (2015) Impact of a structured report template on the quality of mri reports for rectal cancer staging. Am JRoentgenol 205:584-588. https://doi.org/10 2214/AJR.14.14053

23. Schoeppe F, Sommer WH, Norenberg D et al (2018) Structured reporting adds clinical value in primary CT staging of diffuse large B-cell lymphoma. Eur Radiol 28:3702-3709. https://doi.org/10.1007/ s00330-018-5340-3

24. Schwartz LH, Panicek DM, Berk AR et al (2011) Improving communication of diagnostic radiology findings through structured reporting. Radiology 260:174-181. https://doi.org/10.1148/radiol. 11101913

25. Sigl B, Herold C (2021) Strukturierte Befundung in der Radiologie - Chance für die radiologische Jugend? Radiologe 61:487-489. https://doi.org/ 10.1007/S00117-021-00826-2

26. Simpson S, Kay FU, Abbara S et al (2020) Radiological society of North America expert consensus document on reporting chest $\mathrm{CT}$ findings related to COVID-19: endorsed by the Society of Thoracic Radiology, the American College of Radiology, and RSNA. Radiol Cardiothorac Imaging 2:e200152. https://doi.org/10.1148/ryct.2020200152

27. Weiss DL, Langlotz CP (2008) Structured reporting: patient care enhancement or productivity nightmare? Radiology 249:739-747. https://doi.org/10. 1148/radiol.2493080988

28. Wildman-Tobriner B, Allen BC, Bashir MR et al (2017) Structured reporting of CT enterography for inflammatory bowel disease: effect on key feature reporting, accuracy across training levels, and subjective assessment of disease by referring physicians. Abdom Radiol 42:2243-2250. https:// doi.org/10.1007/s00261-017-1136-1

29. PI-RADS | American College of Radiology https:// www.acr.org/Clinical-Resources/Reporting-andData-Systems/PI-RADS.Zugegriffen: 1. Juli 2021

30. Lung Rads|American College of Radiology https:// www.acr.org/Clinical-Resources/Reporting-andData-Systems/Lung-Rads.Zugegriffen:1.Juli2021

31. CT/MRI LI-RADS v2018 | American College of Radiology https://www.acr.org/Clinical-Resources/ Reporting-and-Data-Systems/LI-RADS/CT-MRILI-RADS-v2018.Zugegriffen:30. Juni 2021

\section{Integration of structured reporting into the routine radiological workflow}

Clinical issue: Structured reporting has been one of the most discussed topics in radiology for years. Currently, there is a lack of user-friendly software solutions that are integrated into the IT infrastructure of hospitals and practices to allow efficient data entry.

Standard radiological methods: Radiological reports are mostly generated as free text documents, either dictated via speech recognition systems or typed. In addition, text components are used to create reports of normal findings that can be further edited and complemented by free text.

Methodological innovations: Software-based reporting systems can combine speech recognition systems with radiological reporting templates in the form of interactive decision trees. A technical integration into RIS ("radiological information system"), PACS ("picture archiving and communication system"), and AV ("advanced visualization") systems via application programming interfaces and interoperability standards can enable efficient processes and the generation of machine-readable report data.

Performance: Structured and semantically annotated clinical data collected via the reporting system are immediately available for epidemiological data analysis and continuous Al training.

Evaluation: The use of structured reporting in routine radiological diagnostics involves an initial transition phase. A successful implementation further requires close integration of the technical infrastructure of several systems.

Practical recommendations: By using a hybrid reporting solution, radiological reports with different levels of structure can be generated. Clinical questions or procedural information can be semi-automatically transferred, thereby eliminating avoidable errors and increasing productivity

\section{Keywords}

Reporting systems · Artificial intelligence - Report templates · Structured data acquisition . Decision support 